\title{
Pharmacokinetics and tolerance of dehydroandrographolide succinate injection after intravenous administration in healthy Chinese volunteers
}

\author{
Qian CHEN, Yun LIU, Yan-mei LIU, Gang-yi LIU, Meng-qi ZHANG, Jing-ying JIA, Chuan LU, Chen YU* \\ Central Laboratory, Shanghai Xuhui Central Hospital, Shanghai 200031, China
}

\begin{abstract}
Aim: Dehydroandrographolide succinate (DAS) is extracted from herbal medicine Andrographis paniculata (Burm f) Nees. DAS injection is used in China for the treatment of viral pneumonia and upper respiratory tract infections. The aim of this study is to investigate the pharmacokinetics and tolerance of DAS injection in healthy Chinese volunteers.

Methods: This was a single-center, randomized, single-dose, three-way crossover design study. Nine eligible subjects were randomly divided into 3 groups, and each group sequentially received 80, 160, or $320 \mathrm{mg}$ of DAS infusion according to a three-way Latin square design. Plasma and urine samples were collected and determined using an LC-MS/MS method. Safety and tolerability were determined via clinical evaluation and adverse event monitoring.

Results: For the 80, 160, and $320 \mathrm{mg}$ dose groups, the mean $C_{\max }$ were 4.82, 12.85, and $26.90 \mathrm{mg} / \mathrm{L}$, respectively, and the mean $\mathrm{AUC}_{0-12}$ were $6.18,16.95$, and $40.65 \mathrm{mg} \cdot \mathrm{L}^{-1} \cdot \mathrm{h}$, respectively. DAS was rapidly cleared, with a mean $T_{\max }$ of $0.94-1.0 \mathrm{~h}$ and a $t_{1 / 2}$ of approximately 1.51-1.89 h. Approximately 10.1\%-15.5\% of the intravenous DAS dose was excreted unchanged in urine within $24 \mathrm{~h}$ in the 3 groups, and more than $90 \%$ of unchanged DAS was excreted between 0 and $4 \mathrm{~h}$. The pharmacokinetic profile was similar between male and female subjects. No serious or unexpected adverse events were found during the study, but one mild adverse event (stomachache) was reported.

Conclusion: This study shows that DAS has nonlinear pharmacokinetic characteristics. To guarantee the effective concentration, mulatiple small doses are recommended in clinical regimens.
\end{abstract}

Keywords: dehydroandrographolide succinate; herbal medicine; pharmacokinetics; tolerance; LC-MS/MS analysis

Acta Pharmacologica Sinica (2012) 33: 1332-1336; doi: 10.1038/aps.2012.79; published online 20 Aug 2012

\section{Introduction}

Dehydroandrographolide succinate (DAS, 14-deoxy-11, 12-didehydroandrographolide-3,19-disuccinate, Yanhuning) is obtained by extraction from the well-known herbal medicine Andrographis paniculata (Burm f) Nees, which belongs to the Acanthaceae family and the Andrographis genus (Figure 1). DAS is usually administered through injection after salification with potassium or a combination of potassium and sodium salt ${ }^{[1,2]}$.

In China, DAS injection is widely used for the treatment of viral pneumonia and viral upper respiratory tract infections because of its immunostimulatory, anti-infective and antiinflammatory effect ${ }^{[3-5]}$. It has also been reported that DAS can show anti-atherosclerosis and anti-diabetic nephropathy

\footnotetext{
* To whom correspondence should be addressed.

E-mail clab001@126.com

Received 2011-12-22 Accepted 2012-05-22
}

effects by the regulation of intracellular signaling transduction and clearance of reactive oxygen species ${ }^{[6,7]}$.<smiles>C=C1CCC2C(C)(COC(=O)CCC(=O)O)C(OC(=O)CCC(=O)O)CCC2(C)C1/C=C/C1=CCOC1=O</smiles>

Figure 1. Chemical structure of DAS with the molecular formula $\mathrm{C}_{28} \mathrm{H}_{36} \mathrm{O}_{10}$ and molecular weight 532.59 Da. 
Numerous traditional Chinese herbs and extracted drugs have been used in clinical treatment without safety and pharmacokinetic studies in humans, and their appropriate use has been determined based on thousands of years of experience. Injectable DAS is one of these types of drugs, and it is widely used in China. Only a few studies have been conducted on the pharmacokinetics of DAS tablets or injection in rabbit or rat plasma ${ }^{[8-10]}$. Currently, there is no information on human pharmacokinetics. The objectives of this study were to assess the pharmacokinetics, safety, and tolerance of DAS injection in healthy adult subjects and to evaluate the potential pharmacokinetic differences between male and female subjects.

\section{Materials and methods}

This study was approved by the Independent Ethics Committee (IEC) of Shanghai Xuhui Central Hospital. The study was conducted in accordance with the guidelines on good clinical practice recommended by the SFDA of China ${ }^{[11]}$ and with the ethical standards for human experimentation established by the Declaration of Helsinki ${ }^{[12]}$. All of the subjects were informed of the study's aim, procedures, and risks by a clinical investigator. Each subject gave written informed consent to participate in the study.

\section{Subjects}

Healthy, nonsmoking Chinese males and females aged 18 to 40 years with a body mass index (BMI) between 19 and 25 $\mathrm{kg} / \mathrm{m}^{2}$ were recruited. Each subject met the following criteria: (1) no contraindication or sensitization response to DAS or any related drugs; (2) no history of significant cardiac, hepatic, renal, pulmonary, neurologic, gastrointestinal, or hematologic diseases; (3) no drug abuse, alcoholism or smoking in the recent year; (4) normal vital signs, physical examination, ECG and laboratory findings and negative for HIV and hepatitis B/C; and (5) no pregnancy or lactation for female subjects. Volunteers who took any prescription drugs two weeks before or during the study period or any over-the-counter remedies (including nutritional supplements) one week before or during the study period were excluded. Additionally, volunteers who suffered from serious disease, took part in drug trials, or donated blood in the prior three months were excluded.

\section{Drug}

DAS injection was formulated and supplied by Chongqing Yaoyou Pharma Co, Ltd (Chongqing, China). It is a yellowish powder, and each vial contained $80 \mathrm{mg}$ of DAS (lot number 09081530).

\section{Study design}

This was a single-center, randomized, three-way crossover design study. Using a computer-generated table of random numbers provided by a statistician, nine eligible subjects were randomly divided into three groups and intravenously administered 80, 160, and $320 \mathrm{mg}$ of DAS, respectively, in three treatment periods. Each subject received three intravenous doses of DAS according to the order of the three-way Latin square design (Table 1). The interval between two treatment periods was more than 7 half-lives.

Table 1. Dose of intravenous infusion of DAS injection received in 9 subjects during 3 treatment period

\begin{tabular}{llrrr}
\hline Group & $n$ & Period 1 & Period 2 & Period 3 \\
\hline 1 & 3 & $80 \mathrm{mg}$ & $160 \mathrm{mg}$ & $320 \mathrm{mg}$ \\
2 & 3 & $160 \mathrm{mg}$ & $320 \mathrm{mg}$ & $80 \mathrm{mg}$ \\
3 & 3 & $320 \mathrm{mg}$ & $80 \mathrm{mg}$ & $160 \mathrm{mg}$ \\
\hline
\end{tabular}

For intravenous infusion, DAS was diluted in $250 \mathrm{~mL}$ of a $5 \%$ glucose injection and infused using a pump at a constant rate of $250 \mathrm{~mL}$ per $60 \mathrm{~min}$.

\section{Sample collection}

Plasma and urine samples were collected and analyzed for DAS concentrations. Blood samples $(3 \mathrm{~mL})$ and plasma were collected predose and at $0.167,0.33,0.5,1,1.167,1.333,1.5,2.0$, $2.5,3.0,4.0,6.0,8.0,10.0$, and $12.0 \mathrm{~h}$ after dosing in each treatment period. The collected blood samples were centrifuged at $1500 \times g$ for $5 \mathrm{~min}$ at room temperature $\left(25 \pm 5^{\circ} \mathrm{C}\right)$ within $30 \mathrm{~min}$ of the collection time. The separated plasma was stored below $-30^{\circ} \mathrm{C}$. Urine for the DAS assay was collected predose and 0-4, $4-8,8-12$, and 12-24 h after dosing in each treatment period. After the total volume of urine for each time range was measured, $6 \mathrm{~mL}$ was stored below $-30^{\circ} \mathrm{C}$ until analysis were conducted.

\section{Drug analysis}

A liquid chromatography tandem mass spectrometry (LC-MS/ MS) method for determining DAS in human plasma and urine was established and validated ${ }^{[13]}$. The analytical method for the plasma consisted of the following steps: $50 \mu \mathrm{L}$ of human plasma, $5 \mu \mathrm{L}$ of internal standard (IS) working solution, and $100 \mu \mathrm{L}$ of precipitant (acetonitrile/methanol, 90:10, $v / v$ ) were mixed by shaking in a $1.5-\mathrm{mL}$ polypropylene tube for $10 \mathrm{~s}$ and centrifuged at $15000 \times g$ for $3 \mathrm{~min}$. In total, $50 \mu \mathrm{L}$ of the supernatant was diluted with $450 \mu \mathrm{L}$ water/acetonitrile (60:40, $v / v)$ in sample vials, and $2 \mu \mathrm{L}$ of the dilution was injected to LC-MS/MS. The analytical method for the determination of DAS in human urine involved sample dilution and direct injection into the high-pressure liquid chromatography tandem mass spectrometry system (Applied Biosystems API 5500, CA, USA).

The lower limit of quantitation for the plasma and urine assay was $10 \mathrm{ng} / \mathrm{mL}$, and the linear calibration range was 10-5000 ng/mL. Chromatographic separation was achieved on a Shiseido Capcell C18 MG III column $(100 \mathrm{~mm} \times 2.0 \mathrm{~mm}$ id, $5 \mu \mathrm{m})$, which was preceded by a Phenomenex C18 guard column ( $4 \mathrm{~mm} \times 3 \mathrm{~mm}$ id, $5 \mu \mathrm{m}$ particle size). The mobile phase consisted of $50 / 50(v / v \%)$ acetonitrile and water (containing $0.02 \%$ formic acid), and the flow rate was $0.3 \mathrm{~mL} / \mathrm{min}$. The 
mass transitions were 531.2-99.0 ( $\mathrm{m} / \mathrm{z})$ for DAS and 267.0-252.1 $(\mathrm{m} / \mathrm{z})$ for the IS.

Precision and accuracy were evaluated with six replicates of quality control (QC) samples at four different concentrations $(10,30,500$, and $4000 \mathrm{ng} / \mathrm{mL})$ with three consecutive runs. The interday and intraday accuracy of the plasma and urine quality control samples were $95.3 \%-112.6 \%$ of nominal, and the interday and intraday precision were less than $7 \%^{[14]}$.

\section{Pharmacokinetic calculations}

The pharmacokinetic parameters of DAS were estimated using a standard non-compartmental method ${ }^{[14]}$ with Drug and Statistics software, version 2.1 (University of Science and Technology, Hefei, China). Individual serum concentration-time curves were constructed, and $C_{\max }$ and $T_{\max }$ were obtained by inspection of the plasma concentration data. $\mathrm{AUC}_{0-12}$ was calculated using the linear trapezoidal method for ascending concentrations and the log trapezoidal method for descending concentrations. $\mathrm{AUC}_{0-\infty}$ was calculated as $\mathrm{AUC}_{0-\infty}=\mathrm{AUC}_{0-12}+\mathrm{C}_{12} / k_{\mathrm{e}}$, where $\mathrm{C}_{12}$ represented the last measurable concentration and $\mathrm{k}_{\mathrm{e}}$ was the slope of the linear regression of the natural logarithm-transformed (ln) plasma concentration-time curve in the terminal phase. The plasma elimination half-life $\left(t_{1 / 2}\right)$ was calculated using the equation $t_{1 / 2}=\ln 2 / k_{\mathrm{e}}^{[14]}$.

DAS urine concentrations, urine volumes from individual collection intervals, and nominal times of collection intervals were used to calculate urinary pharmacokinetic parameters. The amount of DAS excreted unchanged in the urine in each collection interval was determined by the product of the urine concentration and the urine volume. $\mathrm{Ae}_{0-24}$ is the sum of DAS collected over all collection intervals $(0$ to $24 \mathrm{~h})$. The percentage of DAS dose recovered in the urine (urine recovery, $\left.\mathrm{Ae} \%_{0-24}\right)$ in $24 \mathrm{~h}$ was calculated as $\mathrm{Ae}_{0-24}$ divided by the administered dose and multiplied by 100 .

\section{Safety and tolerance}

Safety and tolerance were determined by clinical evaluation, including physical examinations, vital signs (body temperature, blood pressure, heart rate, and breathing rate), 12-lead electrocardiograms and laboratory measurements (hematology, serum chemistry, and urinalysis). Adverse events (AEs) were monitored throughout the study. All of the AEs were recorded and evaluated in terms of intensity (mild, moderate, or severe), duration, severity, outcome, and relationship to study drug. Serious adverse events (SAEs) were defined as death or life-threatening, led to disability, required hospitalization, or required medical intervention to prevent permanent impairment or damage.

\section{Statistical analysis}

DAS pharmacokinetic parameters (AUC, $C_{\max }, T_{\max }$, and CLz) were analyzed using an analysis of variance (ANOVA) model appropriate for the variances among dose groups. DAS pharmacokinetic parameter values between female and male subjects were analyzed using a $t$-test. Descriptive statistics were used to summarize demographic data and assess safety variables, including AEs, laboratory assays, vital signs and electrocardiograms. Adverse events are presented as reported frequencies and percentages.

\section{Results}

\section{Study population}

A total of 9 healthy male $(n=6)$ and female $(n=3)$ volunteers were enrolled in the study. All of the subjects were nonsmokers, with a mean age of 24.3 years (range: $21.3-29$ years), a mean weight of $57.4 \mathrm{~kg}$ (range: $49.0-69.8 \mathrm{~kg}$ ) and a mean BMI of 21.1 (range: 19.1-24.0 kg/ $\mathrm{m}^{2}$ ). All of the participants completed the study as planned.

\section{Pharmacokinetics}

The mean plasma DAS concentration versus time profile is presented in Figure 2. The principal pharmacokinetic parameters of DAS are summarized in Table 2. Mean values of the time to peak plasma concentration $\left(T_{\max }\right)$ of the three dose groups ranged from 0.94 to $1.0 \mathrm{~h}$. Plasma DAS concentrations declined rapidly, with short mean elimination halflives of approximately $1.51-1.89 \mathrm{~h}$. For the 80, 160, and 320

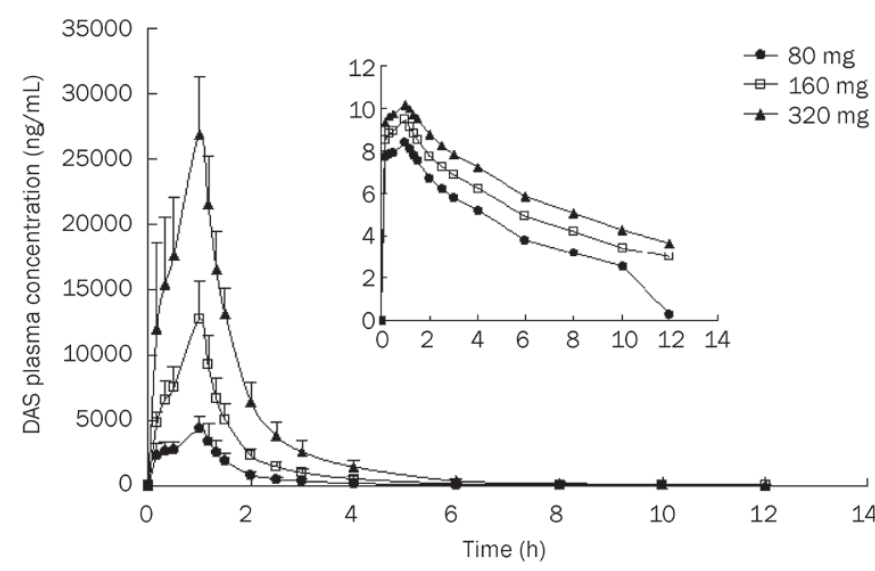

Figure 2. Mean plasma concentrations versus time profiles following single-dose intravenous administration of 80-320 mg DAS injection in Chinese healthy subjects.

Table 2. Pharmacokinetics parameters of DAS (mean \pm SD) following intravenous administration of DAS injection in healthy Chinese subjects $(n=9) .{ }^{\circ} P<0.01$ vs $80 \mathrm{mg}$.

\begin{tabular}{lccc}
\hline Parameters & $80 \mathrm{mg}$ & $160 \mathrm{mg}$ & $320 \mathrm{mg}$ \\
\hline $\mathrm{AUC}_{0-12}\left(\mathrm{mg} \cdot \mathrm{L}^{-1} \cdot \mathrm{h}\right)$ & $6.18 \pm 1.09$ & $16.95 \pm 2.48^{\mathrm{c}}$ & $40.65 \pm 7.53^{\mathrm{c}}$ \\
$\mathrm{AUC}_{0-\infty}\left(\mathrm{mg} \mathrm{L}^{-1} \cdot \mathrm{h}\right)$ & $6.21 \pm 1.10$ & $16.99 \pm 2.49^{\mathrm{c}}$ & $40.74 \pm 7.53^{\mathrm{c}}$ \\
$t_{1 / 2}(\mathrm{~h})$ & $1.51 \pm 0.37$ & $1.80 \pm 0.26$ & $1.89 \pm 0.34$ \\
$T_{\max }(\mathrm{h})$ & $0.94 \pm 0.30$ & $0.94 \pm 0.17$ & $1.00 \pm 0.00$ \\
$C_{\max }(\mathrm{mg} / \mathrm{L})$ & $4.82 \pm 0.96$ & $12.85 \pm 2.76^{\mathrm{c}}$ & $26.90 \pm 4.37^{\circ}$ \\
$\mathrm{MRT}_{0-12}(\mathrm{~h})$ & $1.41 \pm 0.13$ & $1.48 \pm 0.14$ & $1.54 \pm 0.13$ \\
$\mathrm{CLz}(\mathrm{L} / \mathrm{h})$ & $13.27 \pm 2.42$ & $9.60 \pm 1.40^{\mathrm{c}}$ & $8.07 \pm 1.35^{\mathrm{c}}$ \\
$\mathrm{Ae}_{0-24}(\%)$ & $12.33 \pm 3.42$ & $15.56 \pm 2.41$ & $10.17 \pm 2.74$ \\
\hline
\end{tabular}


mg dose groups, mean $C_{\max }$ values were 4.82, 12.85, and 26.90 $\mathrm{mg} / \mathrm{L}$, respectively; mean $\mathrm{AUC}_{0-12}$ values were 6.18, 16.95, and $40.65 \mathrm{mg} \cdot \mathrm{L}^{-1} \cdot \mathrm{h}^{-1}$, respectively; and mean $\mathrm{AUC}_{0-\infty}$ values were $6.21,16.99$, and $40.74 \mathrm{mg} \cdot \mathrm{L}^{-1} \cdot \mathrm{h}^{-1}$, respectively. The AUC and $C_{\max }$ of plasma DAS increased disproportionately to dose, while the clearance rate $(13.27,9.60$, and $8.07 \mathrm{~mL} / \mathrm{min}$, respectively) decreased as the dose increased in the 80, 160, and $320 \mathrm{mg}$ dose groups. The standardized $C_{\max }$ and AUC values of the $80 \mathrm{mg}$ group were statistically significantly different $(P<0.01)$ compared with the other dose groups (Table 3). Approximately $10.1 \%-15.5 \%$ of the intravenous DAS dose was excreted unchanged in urine within $24 \mathrm{~h}$, and more than $90 \%$ of unchanged DAS was excreted from 0 to $4 \mathrm{~h}$. Statistical comparisons indicated that none of the pharmacokinetic parameters were significantly different between female and male subjects (Table 4).

Table 3. The standardized pharmacokinetics parameters of DAS (mean \pm SD) based on $80 \mathrm{mg}$ following intravenous administration of DAS injection in healthy Chinese subjects $(n=9)$. ${ }^{\circ} P<0.01$ vs $80 \mathrm{mg}$.

\begin{tabular}{lrcr}
\hline Parameters & $80 \mathrm{mg}$ & $160 \mathrm{mg}$ & $320 \mathrm{mg}$ \\
\hline $\mathrm{AUC}_{0-12}\left(\mu \mathrm{g} \cdot \mathrm{L}^{-1} \cdot \mathrm{h}\right)$ & $6.18 \pm 1.09$ & $8.48 \pm 1.24^{\mathrm{c}}$ & $10.16 \pm 1.88^{\mathrm{c}}$ \\
$\mathrm{AUC}_{0-\infty}\left(\mu \mathrm{g} \cdot \mathrm{L}^{-1} \cdot \mathrm{h}\right)$ & $6.21 \pm 1.10$ & $8.49 \pm 1.25^{\mathrm{c}}$ & $10.19 \pm 1.88^{\mathrm{c}}$ \\
$\mathrm{C}_{\max }(\mu \mathrm{g} / \mathrm{L})$ & $4.82 \pm 0.96$ & $6.42 \pm 1.38^{\mathrm{c}}$ & $6.72 \pm 1.09^{\mathrm{c}}$ \\
$\mathrm{CLz}(\mathrm{L} / \mathrm{h})$ & $13.27 \pm 2.42$ & $4.80 \pm 0.70^{\mathrm{c}}$ & $2.02 \pm 0.34^{\mathrm{c}}$ \\
\hline
\end{tabular}

\section{Safety and tolerance}

One mild adverse event was reported during the study. Subject $06 \mathrm{had}$ a stomach ache $24 \mathrm{~h}$ post-dose $(320 \mathrm{mg}$ ) in period 3 , which resolved spontaneously in $2 \mathrm{~h}$. No serious adverse events were reported. No clinically significant changes in laboratory values, vital signs, or electrocardiogram safety parameters were observed.

\section{Discussion}

In this study, pharmacokinetic parameters were not proportional to dose. AUC and $C_{\max }$ values increased slightly more compared with the dose among the $80 \mathrm{mg}, 160 \mathrm{mg}$ and 320 mg dose groups. In contrast, the plasma clearance rate was significantly less than dose proportional over the dose range. A similar phenomenon occurred in the rat pharmacokinetic study of DAS and may be attributed to the saturation of metabolic enzymes by the increase in dosage. This hypothesis can also explain the decrease in the clearance rate in the high-dose group. This result can guide clinical prescription to avoid an overdose of DAS and reduce the risk of possible accumulation after drug administration.

Plasma concentrations rapidly decreased from $C_{\max }$, with a $t_{1 / 2}$ of approximately 1.51-1.89 h. Because DAS has a high $C_{\max }$ a short $t_{1 / 2}$ and is less than dose proportional, multiple small doses are recommended. Infusion should be maintained at an appropriate speed to extend the effective period and avoid high dose accumulation. Urine pharmacokinetic data showed that the concentration of DAS in the urine decreased rapidly within $24 \mathrm{~h}$ after drug administration, and more than $90 \%$ of unchanged DAS was excreted within the first $4 \mathrm{~h}$. However, for the 80-, 160-, and 320-mg dose groups, the urinary excretion of the prototype drug in urine were $12.33 \pm 3.42$, $15.56 \pm 2.41$ and $10.17 \pm 2.74$, respectively. These results indicate that DAS injection is rapidly cleared from the plasma, and most DAS may be excreted after being transformed to metabolites.

The pharmacokinetic parameters of DAS were similar in both female and male subjects. However, the limitations of our study are that only a small number of female subjects were enrolled, and more observations are required in a further study to determined gender differences.

In conclusion, DAS injection is safe and well tolerated over the dose range (single doses of DAS injection up to $320 \mathrm{mg}$ ) of intravenous infusion in young, healthy male and female subjects. No serious or unexpected adverse events occurred during the study, and all of the subjects remained in good compliance. AUC and $C_{\max }$ increased slightly more with increasing dosage; however, plasma clearance showed a decreasing trend as the dose increased. DAS is rapidly cleared from the blood, and most of the drug was excreted after transformation to metabolites. This pharmacokinetic study showed that DAS has nonlinear pharmacokinetic characteristics. To guarantee the effective concentration, multiple small doses are recommended in the clinical regimen of DAS.

Table 4. Pharmacokinetics parameters of DAS (mean \pm SD) following intravenous administration of DAS injection in male and female Chinese subjects.

\begin{tabular}{|c|c|c|c|c|c|c|}
\hline Parameters & $\mathrm{M}(n=6)$ & $\mathrm{F}(n=3)$ & $M$ & $\mathrm{~F}$ & M & $\mathrm{F}$ \\
\hline$A \cup C_{0-12}\left(m g \cdot L^{-1} \cdot h\right)$ & $6.02 \pm 1.02$ & $6.51 \pm 1.39$ & $16.37 \pm 2.06$ & $18.11 \pm 3.33$ & $37.79 \pm 4.82$ & $46.36 \pm 9.78$ \\
\hline $\mathrm{AUC}_{0-\infty}\left(\mathrm{mg} \cdot \mathrm{L}^{-1} \cdot \mathrm{h}\right)$ & $6.04 \pm 1.03$ & $6.53 \pm 1.38$ & $16.40 \pm 2.07$ & $18.16 \pm 3.33$ & $37.89 \pm 4.81$ & $46.45 \pm 9.78$ \\
\hline$t_{1 / 2}(\mathrm{~h})$ & $1.57 \pm 0.45$ & $1.40 \pm 0.08$ & $1.74 \pm 0.23$ & $1.93 \pm 0.23$ & $2.04 \pm 0.23$ & $1.59 \pm 0.22$ \\
\hline$T_{\max }(\mathrm{h})$ & $1.03 \pm 0.07$ & $0.78 \pm 0.54$ & $1.00 \pm 0.00$ & $0.83 \pm 0.29$ & $1.00 \pm 0.00$ & $1.00 \pm 0.00$ \\
\hline
\end{tabular}

$\mathrm{M}$, male; $\mathrm{F}$, female. 


\section{Acknowledgements}

We acknowledge Yaoyou Pharma Co, Ltd (Chongqing, China) for providing dehydroandrographolide injection for the study and Wei WANG (Shanghai Xuhui Central Hospital, Shanghai, China) for her help in ECG reporting and clinical observation analysis.

\section{Author contribution}

Yan-mei LIU, Yun LIU and Chen YU designed the research; Yun LIU, Yan-mei LIU and Jing-ying JIA conducted the research; Gang-yi LIU, Meng-qi ZHANG and Chuan LU measured the concentration of DAS; Qian CHEN and Yun LIU analyzed data; Qian CHEN wrote the paper; and Chen YU critically revised the manuscript.

\section{References}

1 Pfisterer PH, Rollinger JM, Schyschka L, Rudy A, Vollmar AM, Stuppner $\mathrm{H}$. Neoandrographolide from Andrographis paniculata as a potential natural chemosensitizer. Planta Med 2010; 76: 1698-700.

2 Liu XW, Fang Y, Wang Q, Li R, Tan JJ, Chao RB. Identification of main related substances in potassium sodium dehydroandrographolide succinate. Yao Xue Xue Bao 2010; 45: 641-6.

3 Akbar S. Andrographis paniculata: a review of pharmacological activities and clinical effects. Altern Med Rev 2011; 16: 66-77.

4 Deng WL, Liu JY, Nie RJ. Pharmacological studies on 14-deoxy-11, 12-didehydroandrographolide-3, 19-disuccinate. I. Anti-inflammatory activity (author's transI)]. Yao Xue Xue Bao 1980; 15: 590-7.

5 Chao WW, Lin BF. Isolation and identification of bioactive compounds in Andrographis paniculata (Chuanxinlian). Chin Med 2010; 13: 17.

6 Lee MJ, Rao YK, Chen K, Lee YC, Chung YS, Tzeng YM. Andrographolide and 14-deoxy-11,12-didehydroandrographolide from Andrographis paniculata attenuate. J Ethnopharmacol 2010; 132:
497-505.

7 Parichatikanond W, Suthisisang C, Dhepakson P, Herunsalee A. Study of anti-inflammatory activities of the pure compounds from Andrographis paniculata (burm.f.) Nees and their effects on gene expression. Int Immunopharmacol 2010; 10: 1361-73.

$8 \mathrm{Xu}$ XQ, Hu GL, Shen JC, Li Q, Wang XR. Determination of andrographolide and dehydroandrographolide in Andrographis paniculata nees materials and related patent medicines by reversed-phase high performance liquid chromatography. Se Pu 2002; 20: 446-8.

9 Han FM, Cai WT, Xia QS, Chen Y. Pharmacokinetics of dehydroandrographolide of Chuanxinlian Tablet in rat. China Journal of Traditional Chinese Medicine and Pharmacy 2005; 20: 206-9.

10 Zhang ZY, Liao GT, Wang BN. Pharmacoinetics study on monopotassium salt of 14-deoxy-11,12-didehdroandographolide-3,19disuccinate (DAS-K). West China Journal of Pharmaceutical Sciences 1991; 6: 129-31.

11 State Food and Drug Administration of China. Guideline for Good Clinical Principles. Available at http://www.sda.gov.cn/WS01/ CL0053/24473.html. Accessed August 6, 2003.

12 World Medical Association Declaration of Helsinki (WMA). Ethical Principles for Medical Research Involving Human Subjects. Adopted by the 18th WMA General Assembly, Helsinki, Finland, June 1964, and amended by the 52nd WMA General Assembly, Edinburgh, Scotland, October 7, 2000. Available at http://www.wma.net/e/ policy/b3.htm. Accessed September 20, 2007.

13 Li SJ, Yang D, Zhang M, Zhou J, Li R, Lu C, et al. Determination of dehydroandrographolide succinate in human plasma by liquid chromatography tandem mass spectrometry (LC-MS/MS): Method development, validation and clinical pharmacokinetic study. J Chromatogr B Analyt Technol Biomed Life Sci 2010; 878: 2274-9.

14 Center for Drug Evaluation, State Food and Drug Administration of China. Guidance for clinical pharmacokinetics studies of chemical drug. Available at http://www.cde.org.cn/zdyz. do?method=largePage\&id=2070. Accessed March, 2005. 